\title{
Improving the Transduction of Bone Marrow-Derived Cells with an Integrase-Defective Lentiviral Vector
}

\author{
S. Louise Pay, ${ }^{1,2}$ Xiaoping Qi, ${ }^{2,3}$ Jeffrey F. Willard, Juliana Godoy, ${ }^{2,3}$ Kavya Sankhavaram, \\ Ranier Horton, ${ }^{2}$ Sayak K. Mitter, ${ }^{2,3}$ Judith L. Quigley, Lung-Ji Chang, \\ Maria B. Grant, ${ }^{2,3}$ and Michael E. Boulton ${ }^{2,3, *}$ \\ ${ }^{1}$ Department of Medical and Molecular Genetics and ${ }^{2}$ Department of Ophthalmology, Eugene and Marilyn Glick Eye Institute, Indiana University School of Medicine, \\ Indianapolis, Indiana; ${ }^{3}$ Department of Ophthalmology, University of Alabama at Birmingham, Birmingham, Alabama; ${ }^{4}$ Department of Molecular Genetics \\ and Microbiology, University of Florida, Gainesville, Florida.
}

In lentiviral vector (LV) applications where transient transgene expression is sufficient, integrase-defective lentiviral vectors (IDLVs) are beneficial for reducing the potential for off-target effects associated with insertional mutagenesis. It was previously demonstrated that human RPE65 mRNA expression from an integrating lentiviral vector (ILV) induces endogenous Rpe65 and Cralbp mRNA expression in murine bone marrowderived cells (BMDCs), initiating programming of the cells to retinal pigment epithelium (RPE)-like cells. These cells regenerate RPE in retinal degeneration models when injected systemically. As transient expression of RPE65 is sufficient to activate endogenous RPE-associated genes for programming BMDCs, use of an ILV is an unnecessary risk. In this study, an IDLV expressing RPE65 (IDLV3-RPE65) was generated. Transduction with IDLV3-RPE65 is less efficient than the integrating vector (ILV3-RPE65). Therefore, IDLV3-RPE65 transduction was enhanced with a combination of preloading $20 \times$-concentrated viral supernatant on RetroNectin at a multiplicity of infection of 50 and transduction of BMDCs by low-speed centrifugation. RPE65 mRNA levels increased from $\sim 12$-fold to $\sim 25$-fold $(p<0.05)$ after modification of the IDLV3-RPE65 transduction protocol, achieving expression similar to the $\sim 27$-fold $(p<0.05)$ increase observed with ILV3RPE65. Additionally, the study shows that the same preparation of RetroNectin can be used to coat up to three wells with no reduction in transduction. Critically, IDLV3-RPE65 transduction initiates endogenous Rpe65 mRNA expression in murine BMDCs and Cralbp/CRALBP mRNA in both murine and human BMDCs, similar to expression observed in ILV3-RPE65-transduced cells. Systemic administration of ILV3RPE65 or IDLV3-RPE65 programmed BMDCs in a mouse model of retinal degeneration is sufficient to retain visual function and reduce retinal degeneration compared to mice receiving no treatment or naïve BMDC. It is concluded that IDLV3-RPE65 is appropriate for programming BMDCs to RPE-like cells.

Keywords: bone marrow-derived cells, lentiviral vectors, transduction efficiency, integrase-defective lentivirus, age-related macular degeneration, retinal pigment epithelium

\section{INTRODUCTION}

WE HAVE PREVIOUSLY DEMONSTRATED that systemic delivery of immature bone marrow-derived cells (BMDC; Lin-/Sca1+), programmed ex vivo by inserting a stable RPE65 transgene using an integrating lentiviral vector (LV), can regenerate an efficient and functional retinal pigment epithelial
(RPE) cell layer that restores visual function in mouse models of retinal degeneration. ${ }^{1,2}$ This has significant implications for the treatment of dry age-related macular degeneration (AMD), which is a major cause of vision loss in the elderly. ${ }^{3}$ The primary defect in dry AMD is believed to be at the RPE, which shows cellular dysfunction, atrophy,

${ }^{*}$ Correspondence: Prof. Michael E. Boulton, Department of Ophthalmology, University of Alabama at Birmingham, 1720 2nd Ave S, VH 476 A, Birmingham, AL 35294. E-mail: meboulton@uabmc.edu

(c) S. Louise Pay et al. 2018; Published by Mary Ann Liebert, Inc. This article is available under the Creative Commons License CC-BYNC (http://creativecommons.org/licenses/by-nc/4.0). This license permits non-commercial use, distribution and reproduction in any medium, provided the original work is properly cited. Permission only needs to be obtained for commercial use and can be done via RightsLink. 
and cell loss, particularly in the central retina. ${ }^{4,5}$ The most viable and attractive option for targeting dry AMD is in the early stages by replacement of damaged RPE using a minimally invasive approach, such as systemic delivery of programmed BMDC, as has been shown in animal models. ${ }^{1,2}$ As success has been observed in murine models with murine cells, it is necessary to optimize and further develop the BMDC programming technique for safe and efficient application in human cells.

It has previously been found that the expression of the RPE65 transgene activates expression of adenylate cyclase in BMDCs within hours of transduction, ${ }^{2}$ and this in turn switches on expression of endogenous Rpe65 and RPE-associated marker Cellular Retinaldehyde-Binding Protein-1 $($ Cralbp $){ }^{2}$ It was therefore hypothesized that transient expression of RPE65 from an integrasedefective lentiviral vector (IDLV) may result in sufficient programming in the absence of integration. Limiting integration significantly reduces the risk of insertional mutagenesis, which can result in deleterious effects resulting from LV insertion in coding regions or regulatory elements. ${ }^{6-10}$ This vector would be ideal for programming BMDCs for use in human clinical trials.

IDLVs are known to be less efficient than integrating lentiviral vectors (ILVs) for a number of reasons, including low transduction rates and episomal silencing. ${ }^{6,7,11}$ Silencing post transduction is a significant concern for long-term expression of a transgene from IDLVs in nondividing cells. ${ }^{11}$ For this application, however, short-term expression of RPE65 is thought to be sufficient for the programming of BMDCs, as it has been shown that cells express the endogenous Rpe65 and Cralbp mRNAs within hours of transduction with the integrating RPE65 vector. ${ }^{1,2}$ This study therefore focused on improving the protocol for transducing BMDCs with IDLV-RPE65 to maximize the number of cells transduced with the vector.

The study demonstrated that IDLV3-RPE65 transduction can be increased by: (1) increasing the concentration of the viral supernatant to reduce the volume required to obtain a high multiplicity of infection (MOI), (2) preloading viral supernatant on RetroNectin prior to transduction, and (3) using a spinoculation method to transduce the cells. In the process of optimizing the protocol IDLV3-RPE65 transduction, measures were included to reduce the costs associated with RetroNectin use for LV transduction by: (1) confirming that using $2 \mu \mathrm{g} / \mathrm{cm}^{2}$ to coat plates is sufficient to achieve transduction with LVs, as has been reported in retroviral vectors ${ }^{12}$; and (2) reusing the $2 \mu \mathrm{g} / \mathrm{cm}^{2}$ preparation to coat up to three wells. IDLV3-RPE65-transduced in this manner induces activation of the endogenous RPE-associated genes Rpe65 and Cralbp in murine BMDCs and $C R A L B P$ in human BMDCs at a similar level to ILV3-RPE65 transduction. This indicates that IDLV3-RPE65 is capable of initiating the differentiation of both mouse and human BMDCs to RPElike cells in vitro, rendering them suitable for therapeutic transplantation in dry AMD.

\section{MATERIALS AND METHODS Plasmids}

A third-generation lentiviral vector expression plasmid, pCDH-EF1-T2A-copGFP, was obtained from Systems Biosciences (CD823A-1). RPE65 gene cDNA was obtained from GeneArt Gene Synthesis Service and cloned into CD832A-1 by Xba1/Sal1 (New England Biolabs; catalog \#R0145S and \#R0138S) digestion of both plasmids and subsequent ligation, placing RPE65 in frame with the EF1 promoter and removing the T2A-cop-GFP region of the plasmid (pCDH-RPE65). T4 DNA ligase (New England Biolabs; catalog \#M0202S) was used to ligate the RPE65-containing fragment with CD832A-1 with a 3:1 ratio of insert to vector. Ligations were transformed into TOP10 bacteria, screened, and verified by polymerase chain reaction (PCR). Plasmid minipreps were used to obtain plasmid for sequence verification and in the intermediate stages of vector cloning, obtained using the Qiagen Spin Miniprep Kit (catalog \#27106). Packaging plasmids pMDL and pMDL-D116N, and the helper plasmid Rev, were obtained from Dr. Kenneth Cornetta (IU Vector Production Facility). The envelope plasmid pcz-VSVG was obtained from Dirk Lindemann (Dresden University). Plasmids used for the production of lentiviruses were prepared by maxiprep using the PureLink HiPure Plasmid Filter Maxiprep Kit from Invitrogen (catalog \#K210017) or Qiagen Plasmid Maxi Kit (catalog \#12165). Plasmid DNA concentration and quality was measured using the NanoDrop 2000 Spectrophotometer (Thermo Fisher Scientific; catalog \#SO6497).

\section{Bacterial growth and transformation}

All bacteria were grown in Luria-Bertani (LB) broth ( $\mathrm{pH} 7.5)$ or on LB agar supplemented with $100 \mu \mathrm{g} / \mathrm{mL}$ of ampicillin (Thermo Fisher Scientific; BP1760-25) or $50 \mu \mathrm{g} / \mathrm{mL}$ of kanamycin (Teknova; K2127) as required. Prior to cloning, all plasmids were transformed into dam/dcm-negative Escherichia coli strain JM110 obtained from Agilent (catalog \#200239) due to the presence of methylation-sensitive restriction sites in the 
cloning protocols. After cloning, plasmids were transformed into $E$. coli TOP10 bacteria. pUC18 and sterile $\mathrm{dH}_{2} \mathrm{O}$ were used as positive and negative controls, respectively.

\section{Lentiviral production and titer}

ILV3-RPE65 and IDLV3-RPE65 were produced by co-transfection of viral production plasmids in $10 \mathrm{~cm}$ tissue culture plates containing $8 \times 10^{6} \mathrm{HEK}-$ 293T cells (HEK 293T/17 ATCC-CRL-11268) per plate. DNA mixture for each plate was prepared as follows: $1 \mathrm{~mL}$ of plain Dulbecco's modified Eagle's medium (DMEM) with $5 \mu \mathrm{g}$ of pcz-VSVG, $10 \mu \mathrm{g}$ of pCDH-RPE65 or pCDH-EF1-T2A-copGFP expression vector, $5 \mu \mathrm{g}$ of packaging plasmid pMDL (for ILV3) or pMDL-D116N (for IDLV3), and $1 \mu \mathrm{g}$ of Rev. DNA mixture was added to $955 \mu \mathrm{L}$ of plain DMEM containing $45 \mu \mathrm{L}$ of $1 \mathrm{mg} / \mathrm{mL}$ polyethylenimine (PEI; Sigma-Aldrich; catalog \#900298-6) and incubated at room temperature in the dark for $20 \mathrm{~min}$. The DNA/PEI mixture for each sample was then added to each plate containing HEK-293T cells and incubated for $16 \mathrm{~h}$ at $37^{\circ} \mathrm{C}$. The medium was replaced with $5 \mathrm{~mL}$ of fresh complete DMEM, and the plates were incubated for $24 \mathrm{~h}$. Viral supernatant was harvested and filtered through a $0.45 \mu \mathrm{m}$ polyethersulfone membrane (Thermo Fish Scientific; Nalgene Filter Unit; catalog \#166-0045) and concentrated either by ultracentrifugation at $10,000 \mathrm{~g}$ for $1-4 \mathrm{~h}$ or with Lenti-X Concentrator reagent for $30 \mathrm{~min}$ to a final concentration of $10 \times$ or $20 \times$ as per the instruction manual (ClonTech; catalog \#PT4421-2). Supernatant was re-suspended in phosphate-buffered saline (PBS) or complete DMEM and titered in HT1080 cells.

\section{Calculation of viral titer in the absence of a selection marker}

A standard curve was generated by comparing $2^{-\Delta \Delta \mathrm{CT}}$ values with flow cytometric data for green fluorescent protein positive $\left(\mathrm{GFP}^{+}\right)$cells in order to allow a comparison between $2^{-\Delta \Delta \mathrm{CT}}$ values and viral titer. A $2^{-\Delta \Delta \mathrm{CT}}$ value of 30 where $10 \mu \mathrm{L}$ of viral supernatant was added per well containing 50,000 cells was considered to represent a viral titer of $10^{8}$ at a MOI of 50 ; where a $2^{-\Delta \Delta \mathrm{CT}}$ value of 30 was obtained with $2.5 \mu \mathrm{L}$ viral supernatant, viral titer was $\sim 10^{9}$ at a MOI of 50 .

\section{Animals and isolation of murine BMDC}

C57BL6/J GFP ${ }^{+}$mice were used for the isolation of Lin-/Sca1+ BMDC, as previously described. ${ }^{1,2}$ Animal procedures were performed according to IACUC protocols \#10568 and \#11155 approved by the IUSM IACUC. All procedures were performed ac- cording to the Declaration of Helsinki and the ARVO regulations for animal use. In brief, immediately after sacrifice, the tibiae and fibiae were isolated, and the bone marrow was flushed out of each bone with PBS $+1 \mathrm{mM}$ of EDTA, filtered through a $100 \mu \mathrm{m}$ mesh cell strainer, re-suspended in PBS + EDTA, and centrifuged at $300 \mathrm{~g}$ (Eppendorf Centrifuge 5804). Supernatant was discarded, and ammonium chloride red blood cell lysis buffer was added. After 15 min on ice, the mixture was centrifuged at $300 \mathrm{~g}$, and the cells were re-suspended in PBS + EDTA at a concentration of $1 \times 10^{8}$ cells $/ \mathrm{mL}$.

\section{Lin-/Sca1+ BMDC selection}

Lineage depletion was carried out using the EasySep Mouse Hematopoietic Progenitor Cell Enrichment Kit (catalog \#19856). Following isolation, cells were re-suspended at a concentration of $1 \times 10^{8}$ cells $/ \mathrm{mL}$ in PBS + EDTA. Sca1 PE Positive Labeling Reagent $(20 \mu \mathrm{L}$; Stemcell Technologies; catalog \#18756) was added per milliliter of cells. After washing, cells were centrifuged at $350 \mathrm{~g}$ for $5 \mathrm{~min}$, washed, and finally re-suspended at a concentration of 10 million cells $/ \mathrm{mL}$ for fluorescent activated cell sorting (FACS). Where $\mathrm{GFP}^{+}$cells were used, cells were sorted for double-positive $\mathrm{GFP}^{+}($cells)/PE (Sca1+), with a 7-12\% recovery. Where wild-type cells were used, cells were sorted for PE (Sca1+), with a $7-12 \%$ recovery. Sorted cells were post sorted to verify purity and were typically $>95 \%$ pure.

\section{Isolation of human CD34+ BMDCs}

Human CD34+ BMDCs were obtained from two sources: (1) CD34+ cells with a purity $>99 \%$ isolated from cord blood, obtained from the Indiana University AngioBiocore; and (2) healthy donors (IRB \#160883855). White blood cells were isolated on a Ficoll gradient followed by FACS for CD34+ cells stained with AlexaFluor488 anti-human CD34 (BioLegend; catalog \#344518) or bead separation (Stemcell Technologies; EasySep Human CD34 Positive Selection Kit; catalog \#18056).

\section{LV transduction of cell lines and primary cells}

Transductions were carried out using a number of different methods to identify the optimum procedure. Briefly, BMDCs were transduced overnight for $16 \mathrm{~h}$ or by $2 \mathrm{~h}$ of spinoculation at $150 \mathrm{~g}$ at $21^{\circ} \mathrm{C}$, with no reagent, polybrene, protamine, or RetroNectin at a concentration of $0-12 \mu \mathrm{g} / \mathrm{mL}$ added to media, or on RetroNectin at $0-12 \mu \mathrm{g} / \mathrm{cm}^{2}$. RetroNectin was either preloaded with vector for $30 \mathrm{~min}$ at $37^{\circ} \mathrm{C}$ prior to transduction, or cells and vector were added together at the same time as the transduction. RetroNectin was used to coat up to 
four plates per preparation. Cells were transduced at a concentration of $5,000-100,000$ cell $\mathrm{s} / \mathrm{mL}$ at an MOI of 50, unless a higher or lower MOI is indicated. Where polybrene or protamine sulfate was used for in vitro experiments, polybrene was used at a concentration of $8 \mu \mathrm{g} / \mathrm{mL}$, and protamine sulfate was used at a concentration of $10 \mu \mathrm{g} / \mathrm{mL}$. Where RetroNectin plates or tubes were preloaded with either cells or viral particles, preloading was carried out for $30 \mathrm{~min}$ at $37^{\circ} \mathrm{C}$ in the lowest volume of medium required to cover the surface of the plates. Cells were harvested and prepared for RNA analysis $4 \mathrm{~h}$ after transduction.

\section{Quantitative reverse transcription PCR}

The RNAEasy Mini Kit was used for all RNA isolations. cDNA (25 ng) per microliter was reverse transcribed using the iScript kit (BioRad; catalog \#1708891). BioRad SSoFast Advanced Universal Probes Supermix kits (BioRad; catalog \#1725284) were used for all quantitative reverse transcription PCR (qRT-PCR) assays in $10 \mu \mathrm{L}$ reactions. Where available, BioRad PrimePCR validated primers were used for all qRT-PCR experiments. Each sample was prepared in triplicate, and each measurement was made in triplicate to allow for statistical analysis. Positive PCR controls for RPE65 experiments consisted of cDNA prepared from ARPE19 cells; for GFP experiments, positive controls consisted of cDNA prepared from the bone marrow of $\mathrm{GFP}^{+}$mice. Negative controls for qRTPCRs consisted of a no-template control. Negative controls for experiments consisted of untransduced or untreated cells. GAPDH or beta-actin was used as internal controls for all qRT-PCR experiments. Samples were run on a BioRad CFX96 Touch RealTime PCR Detection System (BioRad; catalog \#1855195). The comparative CT $\left(2^{-\Delta \Delta \mathrm{CT}}\right)$ method was used for analyses.

\section{Efficacy of ILV3 and IDLV3 in a murine model of retinal degeneration}

All animal studies were conducted under approved protocols by the Institutional Animal Care and Use Committee at Indian University and in accordance with guidelines set forth by the $\mathrm{Na}$ tional Institutes of Health and the Statement for the Use of Animals in Ophthalmic and Visual Research of the Association for Research in Vision and Ophthalmology. Adult (7- to 10-week-old) female C57BL6/J mice and homozygous GFP transgenic (C57BL/6-Tg [UBC-GFP]) were purchased from Jackson Laboratories. Generation of SOD2 knockdown mice and injection of BMDCs was performed as previously described. ${ }^{1}$ In brief, the right eyes of mice were injected sub-retinally with $1 \mu \mathrm{L}$ of $2.5 \times 10^{12}$ particles $/ \mathrm{mL}$ of recombinant adenoassociated virus serotype 1 (AAV1) constructs expressing SOD2-specific hammerhead ribozyme, or rAAV-inactive ribozyme. At 1 month following SOD2 knockdown, mice received a systemic injection of $5 \times 10^{4}$ null, ILV3-RPE65, or IDLV3-RPE65 programmed BMDCs in $100 \mu \mathrm{L}$ of sterile saline into the tail vein. Animals were then evaluated 3 months later. To evaluate the optokinetic response, a computer-based visual acuity response test was used (OptoMotry ${ }^{\odot}$ 1.7.7 Cerebral Mechanics, Inc.). In brief, mice were tested for visual acuity by observing optokinetic responses of mice to rotating sinusoidal gratings. ${ }^{1}$ The OptoMotry ${ }^{\odot}$ program changed spatial frequency based on the observer's responses and tracked the responses according to direction of pattern rotation to assess the two eyes independently. For optical coherence tomography (OCT), three lateral images (nasal, central, and temporal) were collected, starting $0.4 \mathrm{~mm}$ above the meridian crossing through the center of the optic nerve (ON), at the ON meridian, and $0.4 \mathrm{~mm}$ below the $\mathrm{ON}$ meridian using a Bipotogen SD-OCT. Upon completion of the in vivo analyses, animals were terminated, and eyes were enucleated. For some eyes, flat mount $\mathrm{RPE} /$ choroid preparations were assessed by confocal imaging using an Olympus BX50 confocal microscope to identify $\mathrm{GFP}^{+}$donor cells. ${ }^{1}$ For general histological analysis, eyes were fixed with $4 \%$ paraformaldehyde (Wako) and embedded in paraffin, and $10 \mu \mathrm{m}$ sections were stained with hematoxylin and eosin according to standard protocols.

\section{Statistical analysis}

All experiments were performed at least in triplicate. Results are expressed as mean \pm standard error of the mean (SEM). Analysis of variance with Tukey's post hoc tests were carried out to determine significance of results. Statistical analysis was performed using Prism v5.01 (GraphPad Software, Inc.), with $p<0.05$ considered statistically significant.

\section{RESULTS}

\section{Transduction of BMDCs with third-generation ILV and IDLV vectors}

Transduction of murine BMDCs with ILV3$R P E 65$ at a MOI of 50 resulted in a $\sim 30$-fold $(p<0.05)$ increase in expression of human RPE65 compared with the null control (Fig. 1A). Expression of murine Rpe65 mRNA was increased ap- 

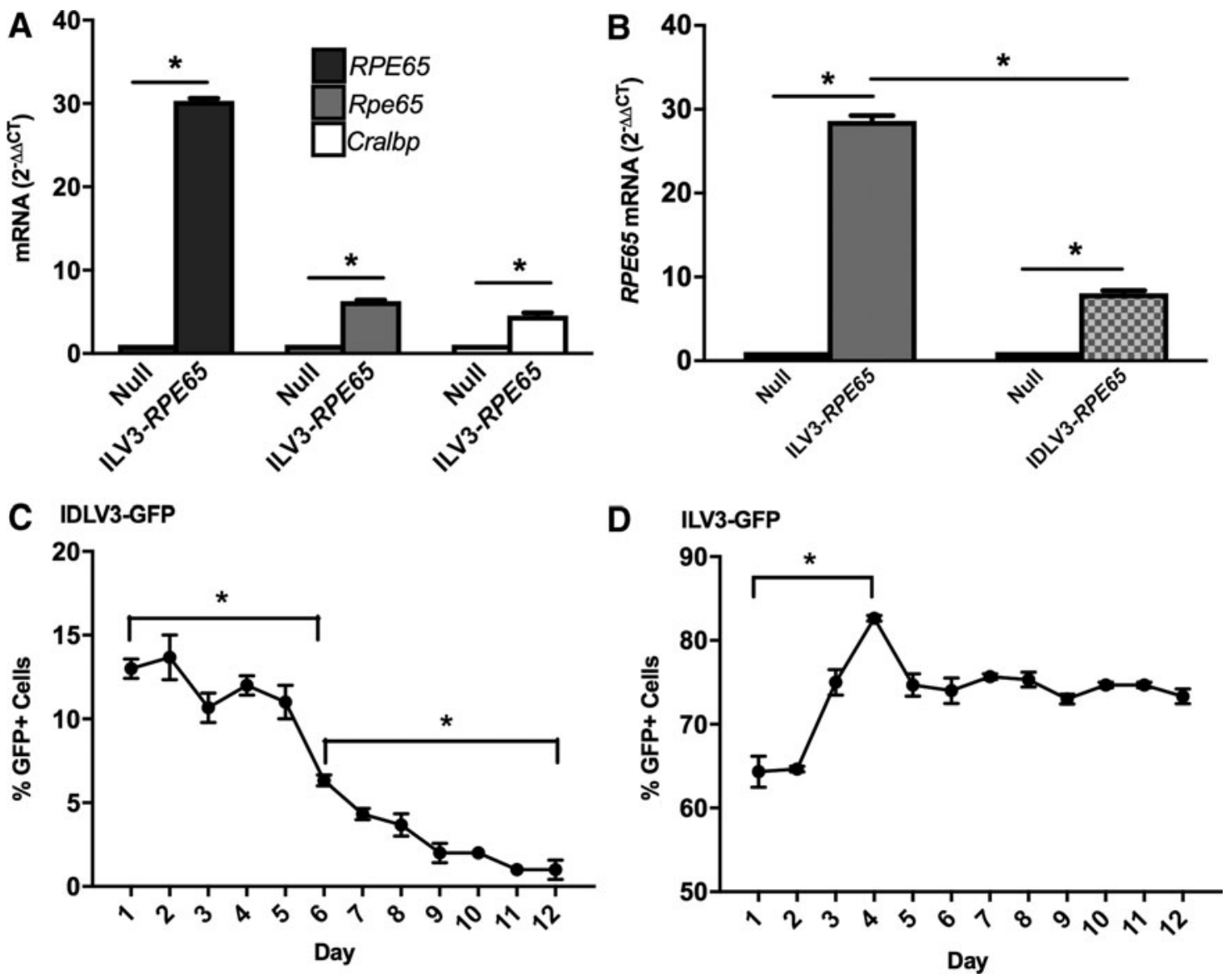

Figure 1. Integrase-defective lentiviral vectors (IDLVs) infect murine bone marrow-derived cells (BMDCs) and induce expression of endogenous mRNA, and transduce HT1080 cells with minimal integration. Murine Lin-/Sca1+ cells were infected with ILV3-RPE65 (A) or IDLV3-RPE65 (B) on RetroNectin by centrifugation for $2 \mathrm{~h}$ at $150 \mathrm{~g}$ prior to harvest for quantitative reverse transcription polymerase chain reaction (qRT-PCR) analysis. HT1080 cells were infected with IDLV3-GFP (C) or ILV3-GFP (D) with polybrene for $16 \mathrm{~h}$ and cultured for 1-12 days with green fluorescent protein (GFP) expression measured each day. Uninfected cells were used as a negative control. For the GFP assays, cells stably expressing GFP were used as a positive control. (A) Expression of RPE65 mRNA from ILV3-RPE65 was $\sim 30$-fold higher than in untreated cells. Cells also expressed the endogenous Rpe65 and Cralbp mRNA approximately six- and fourfold higher than uninfected cells, respectively $(p<0.05)$. $\left(^{*} p<0.05 ; n=9\right)$. (B) Expression of RPE65 mRNA from ILV3-RPE65 was $\sim 28$-fold higher than expression in the negative control $(p<0.05)$. Expression of RPE65 mRNA from IDLV3-RPE65 was approximately eightfold higher than in the negative control $(p<0.05)$. Expression of RPE65 was significantly lower from IDLV3-RPE65 than ILV3-RPE65 $(p>0.05)$. $\left({ }^{*} p<0.05 ; n=3\right)$. (C) The percentage of cells expressing GFP was measured each day. Between day 1 and day 6, expression of GFP reduced from $\sim 13 \%$ to $\sim 6 \%$ ( $p<0.05$ ). Expression further reduced between day 6 and day 12 from $\sim 6 \%$ to $\sim 1 \%(p<0.05)$. $\left.{ }^{*} p<0.05 ; n=3\right)$. (D) The percentage of cells expressing GFP was measured each day. Between day 1 and day 4 , GFP expression increased from $\sim 64 \%$ to $82 \%(p<0.05)$ before stabilizing at $\sim 75 \%$ through day 12 . $\left({ }^{*} p<0.05\right.$ [standard error of the mean (SEM)]; $\left.n=3\right)$. All statistical significance was calculated using one-way analysis of variance (ANOVA) followed by Tukey's multiple comparisons test.

proximately sixfold, and Cralbp approximately fivefold (Fig. 1A). As expression of these endogenous murine mRNAs is a critical step in BMDC programming, ${ }^{1,2}$ it was concluded that ILV3RPE65 is appropriate for use in programming BMDCs and for packaging as an IDLV. The average expression of human RPE65 from ILV3-RPE65 in murine BMDCs was $\sim 28$-fold ( $p<0.05$ ) higher than expression in the untransduced control, and expression of human RPE65 from IDLV3-RPE65 was $\sim 3.6$-fold $(p<0.05)$ lower than expression obtained with ILV3-RPE65 at the same MOI (Fig. 1B). The study therefore focused on improving IDLV3-RPE65 transduction.
To confirm that IDLV3 vectors have limited integration potential, HT1080 cells were transduced with IDLV3-GFP, and the percentage of $\mathrm{GFP}^{+}$cells was monitored for 12 days. This experiment was carried out in a cell line as opposed to in murine or human BMDCs due to previous observations that BMDCs transduced with ILV or IDLV do not survive for sufficient time ex vivo for residual integration to be monitored. Expression of GFP from IDLV3-GFP-transduced cells was significantly decreased $(p<0.05)$ at day 6 in comparison to day 1 , with a 2.1-fold decrease in expression of GFP at day 6 compared with day 1 (Fig. 1C). Expression of GFP was almost absent by day 12 (Fig. 1C), with a 14.9- 
fold decrease in expression compared to day 1 and a 7.2-fold decrease compared to day $6(p<0.05)$. Overall, between day 1 and day 12, the percentage of cells expressing GFP decreased from $\sim 13 \%$ to $\sim 1 \%(p<0.05)$. The residual expression of GFP retained at the end of the experiment is likely a result of a small number of viral integrations, as some residual integration does occur with IDLVs. ${ }^{13}$ In contrast, ILV3-GFP stably integrates into the genome, with expression rising from $\sim 65 \%$ to $\sim 82 \%(p<0.05)$ from day 1 to day 4 before stabilizing at $\sim 75 \%$ from day 5 through day 12 (Fig. 1D). Together, Fig. 1C and D show that transduction with IDLV3-RPE65 or ILV3-RPE65 results in low integration and stable integration, respectively.

\section{Optimization of the use of RetroNectin in the transduction of BMDCs}

The co-localization of cells and LV particles is critical for efficient transduction, and the goal of transduction-promoting reagents such as polycations and the recombinant human fibronectin fragment RetroNectin is to enhance the nonspecific adhesion of the virions to the target cell surface. ${ }^{12,14}$ Previously published experiments were carried out using polybrene. ${ }^{1,2}$ However, as polybrene is known to be toxic to some cell lines and primary cells, first transduction efficiencies were compared with polybrene, the less toxic polycation protamine sulfate, and RetroNectin.

ILV3-RPE65 and IDLV3-RPE65 transduction with protamine sulfate was significantly less efficient than transduction in the presence of polybrene or RetroNectin $(p<0.05$; Fig. $2 \mathrm{~A}$ and B).
Protamine sulfate yielded an $\sim 8$ - and $\sim 11$-fold increase in RPE65 from ILV3-RPE65 in both murine and human BMDCs, respectively, whereas polybrene or RetroNectin yielded a $\sim 25$ - and $\sim 24$ fold increase in ILV3-RPE65-transduced cells $(p<0.05$; Fig. 2A). In IDLV3-RPE65-transduced cells, an approximately five- and fourfold increase in RPE65 was observed with protamine sulfate in murine and human BMDCs, respectively, compared to a $\sim 14$ - and 13-fold increase in the presence of RetroNectin, and $\sim 12$ - and $\sim 13$-fold increase in the presence of polybrene $(p<0.05$; Fig. 2B). Interestingly, combining RetroNectin and polybrene together resulted in a lower transduction efficiency than when each reagent was used individually, with an average fold increase of $\sim 28(p<0.05)$ in RPE65 from ILV3-RPE65 when used individually compared to an average fold increase of $\sim 21.5(p<0.05)$ when used together (Fig. 2C). A similar effect was observed for both murine and human BMDCs with IDLV3-RPE65, with a $\sim 16$-fold $(p<0.05)$ increase in RPE65 with both polybrene and RetroNectin individually compared to a $\sim 13$-fold $(p<0.05)$ increase in expression when used in combination (Fig. 2D).

The transduction efficiency of ILV3-RPE65 and IDLV3-RPE65 vectors on increasing concentrations of RetroNectin was compared to determine the lowest concentration required to yield high levels of transduction. The instruction manual suggests a concentration of $4-20 \mu \mathrm{g} / \mathrm{cm}^{2}$, but it has been reported that cells can be transduced with retroviral vectors on $2 \mu \mathrm{g} / \mathrm{cm}^{2}$ RetroNectin. ${ }^{12}$ A $>25$-fold $(p<0.05)$ increase in RPE65 from ILV3RPE65 in both murine and human BMDCs was

Figure 2. Optimization of the use of RetroNectin for infection of BMDCs. Murine (Lin-/Sca1+) or human (CD34+) BMDCs were infected at a multiplicity of infection (MOI) of 50 with ILV3-RPE65 or IDLV3-RPE65 with polybrene $(8 \mu \mathrm{g} / \mu \mathrm{L})$, protamine sulfate $(10 \mu \mathrm{g} / \mu \mathrm{L})$, or RetroNectin $(2,4$, or $12 \mu \mathrm{g} / \mu \mathrm{L}$; or 2 , 4, or $12 \mu \mathrm{g} /$ $\mathrm{cm}^{2}$ ), or a combination of polybrene and RetroNectin $\left(2 \mu \mathrm{g} / \mathrm{cm}^{2}\right)$. Cells were infected for $12 \mathrm{~h}$ and harvested for qRT-PCR analysis at $16 \mathrm{~h}$. (A) Human RPE65 mRNA levels increased $\sim 25$-fold (murine BMDCs) and $\sim 23$-fold (human BMDCs) with ILV3-RPE65 infection on RetroNectin. Similar levels were observed with polybrene: 24-fold $(p<0.05)$ in the murine model and 23-fold $(p<0.05)$ in the human model. Protamine sulfate was less effective, with around an eightfold $(p<0.05$; murine) and a $\sim 10$-fold ( $p<0.05$; human) increase in human RPE65 mRNA. (B) With IDLV3-RPE65, human RPE65 mRNA increased $\sim 15$-fold ( $p<0.05$; murine) and $\sim 14$-fold ( $p<0.05$; human) with RetroNectin, 13-fold ( $p<0.05$; murine) and 14-fold ( $p<0.05$; human) with polybrene, and around sixfold ( $p<0.05$; murine) and fivefold ( $p<0.05$; human) with protamine sulfate. (C) Human RPE65 mRNA levels were increased $\sim 29$-fold (murine) and $\sim 28$-fold (human) BMDCs infected with RetroNectin, 27-fold ( $p<0.05$; murine) and 28-fold ( $p<0.05$; human) with polybrene, and $\sim 21$-fold $(p<0.05$; murine) and $\sim 20$-fold $(p<0.05$; human) with RetroNectin and polybrene in combination. (D) Human RPE65 mRNA levels were increased $\sim 16$-fold over control in human BMDCs infected with ILDV3-RPE65 using RetroNectin, 16 -fold $(p<0.05)$ with polybrene, and $\sim 13$-fold $(p<0.05)$ with RetroNectin and polybrene in combination. (E) Murine and human BMDCs infected with ILV3-RPE65 in the presence of $2 \mu \mathrm{g} / \mathrm{cm}^{2}$ RetroNectin expressed human RPE65 mRNA mRNA 27- and 28-fold over control ( $p<0.05$ ), respectively. Expression was not increased in either cell type with an increase in RetroNectin concentration to 4 or $12 \mu \mathrm{g} / \mathrm{cm}^{2}$. (F) Murine and human BMDCs infected with IDLV3-RPE65 in the presence of $2 \mu \mathrm{g} / \mathrm{cm}^{2}$ RetroNectin expressed human RPE65 mRNA 13-fold over control $(p<0.05)$ in both cell types. Expression was not increased in either cell type with an increase in RetroNectin concentration to 4 or $12 \mu \mathrm{g} / \mathrm{cm}^{2}$. (G) Murine and human BMDCs infected with ILV3-RPE65 in the presence of $2 \mu \mathrm{g} / \mathrm{cm}^{2}$ RetroNectin used to coat one plate expressed human RPE65 mRNA 23- and 24-fold over control $(p<0.05)$, respectively. Levels did not increase or decrease in cells infected with ILV3-RPE65 in the presence of $2 \mu \mathrm{g} / \mathrm{cm}^{2}$ RetroNectin used to coat two or three plates. In contrast, where $2 \mu \mathrm{g} /$ $\mathrm{cm}^{2}$ RetroNectin was used to coat a fourth plate, RPE65 mRNA levels were only seven- and eightfold over control $(p<0.05)$ in murine and human cells, respectively. (H) Murine and human BMDCs infected with ILV3-RPE65 on $2 \mu \mathrm{g} / \mathrm{cm}^{2}$ RetroNectin-coated plates expressed human RPE65 mRNA 24- and 25-fold over control, respectively $(p<0.05)$. Cells transduced with media containing 2,4 , or $12 \mu \mathrm{g} / \mu \mathrm{L}$ RetroNectin did not significantly express $R P E 65 \mathrm{mRNA}$. ${ }^{*} p<0.05$ [SEM]; $n=3$ ). All statistical significance was calculated using one-way ANOVA followed by Tukey's multiple comparisons test. 

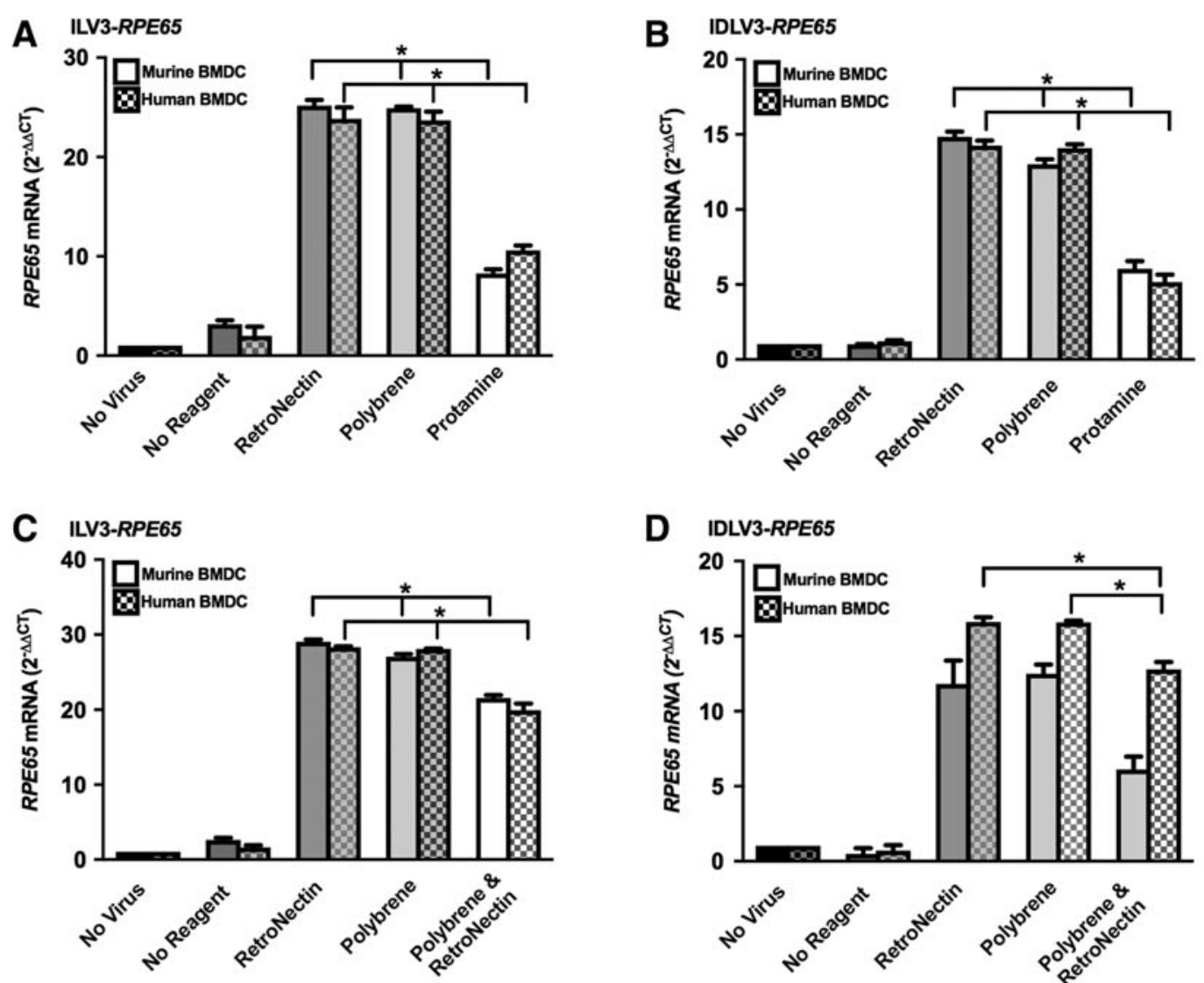

D IDLV3-RPE65
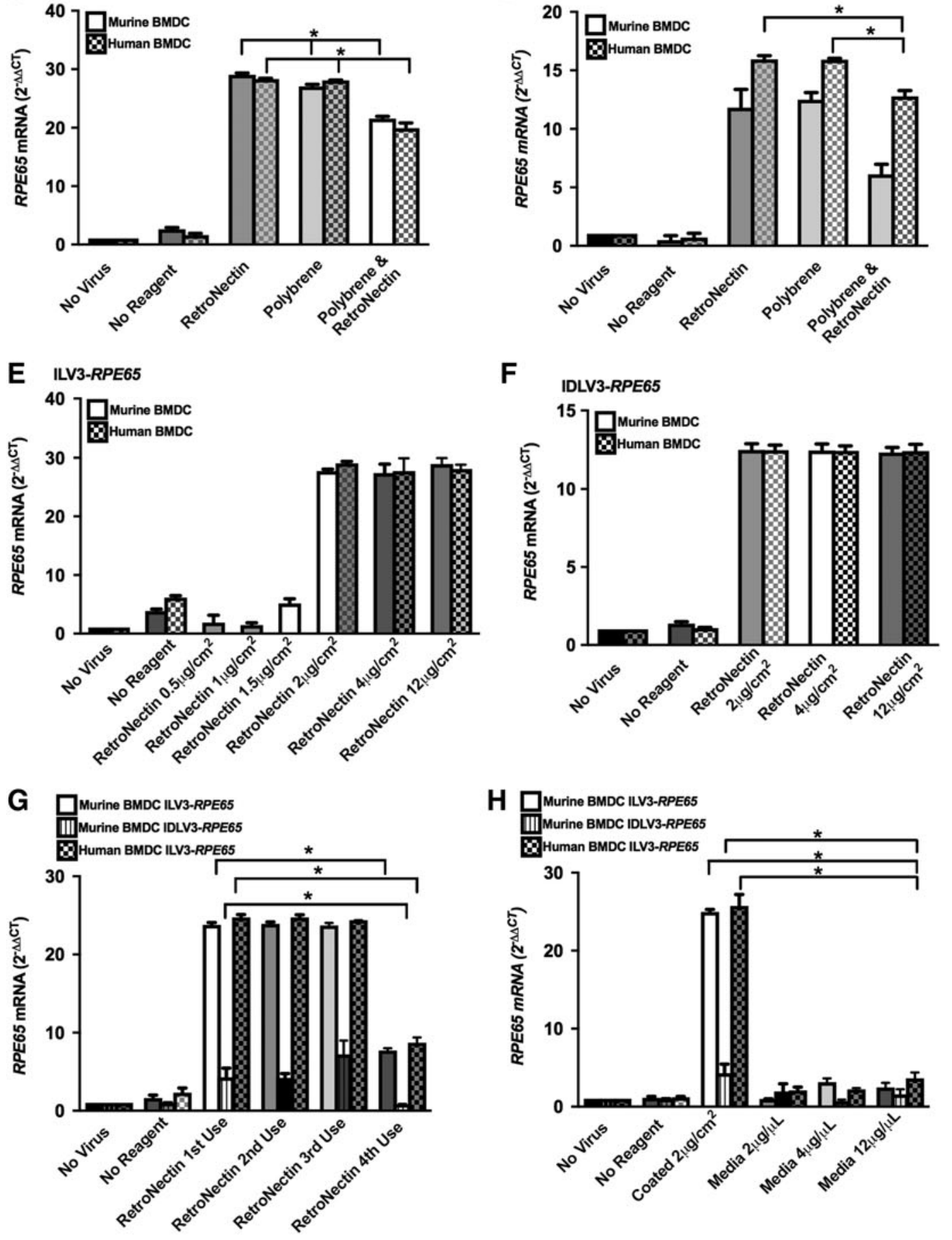
observed when transduced on $2 \mu \mathrm{g} / \mathrm{cm}^{2}$ RetroNectin (Fig. 2E). Transduction was not significantly enhanced by transducing the cells on 4 or $12 \mu \mathrm{g} / \mathrm{cm}^{2}$ RetroNectin (Fig. 2E). Similarly, transduction with the IDLV results in a $\sim 12$-fold $(p<0.05)$ increase in expression in murine and human BMDCs transduced on $2 \mu \mathrm{g} / \mathrm{cm}^{2}$ RetroNectin, which is not significantly enhanced when the concentration of RetroNectin is increased, with a similar fold increase observed when cells are transduced on 4 or $12 \mu \mathrm{g} / \mathrm{cm}^{2}$ RetroNectin (Fig. $2 \mathrm{~F}$ ). It was therefore confirmed that the use of $2 \mu \mathrm{g} / \mathrm{cm}^{2}$ RetroNectin is appropriate for transducing murine and human BMDCs with ILV3-RPE65 and IDLV3RPE65. Using a smaller volume of RetroNectin was not found to facilitate transduction as efficiently as $2 \mu \mathrm{g} / \mathrm{cm}^{2}$ (Fig. 2E).

Next, the binding capacity of RetroNectin when used to coat multiple plates was investigated. Instead of discarding the RetroNectin after coating the first plate, it was transferred to a new plate, and the coating procedure was repeated up to three times. As shown in Fig. 2G, RPE65 expression in both human and murine BMDCs transduced with ILV3-RPE65 was found to be $\sim 27$-fold $(p<0.05)$ higher than in the control in cells transduced on RetroNectin used once, twice, and three times. In contrast, on the fourth use of RetroNectin, RPE65 levels dropped to around eightfold over the control, indicating that RetroNectin cannot be used more than three times (Fig. 2G). Consistent with ILV3-RPE65 data, BMDCs transduced with IDLV3-RPE65 on plates coated with RetroNectin once, twice, three times, or four times expressed RPE65 4-, 4-, 7-, and 0.85-fold over the control, respectively. This further indicates that RetroNectin can be used multiple times prior to a reduction in transduction efficiency (Fig. 2G).

Finally, the study investigated whether adding RetroNectin to the medium could also facilitate transduction. RetroNectin was added to the medium at a concentration of $0-12 \mu \mathrm{g} / \mu \mathrm{L}$, and human RPE65 levels were compared to those achieved on plates coated with $2 \mu \mathrm{g} / \mathrm{cm}^{2}$ RetroNectin following transduction with ILV3-RPE65 or IDLV3-RPE65. Adding RetroNectin to the culture medium does not facilitate ILV3-RPE65 or IDLV3-RPE65 transduction of murine or human BMDCs, with no significant increase in RPE65 expression observed (Fig. $2 \mathrm{H}$ ). By contrast, cells transduced with 2,4 , or $12 \mu \mathrm{g} / \mathrm{mL}$ RetroNectin in the medium were found to express RPE65 $\sim 16, \sim 10$, and $\sim 8$-fold $(p<0.05)$ less, respectively (Fig. $2 \mathrm{H}$ ).

\section{Enhancing the transduction of BMDCs with IDLV3-RPE65}

IDLV3-RPE65 transduces murine and human cells at a lower efficiency than ILV3-RPE65 at the same MOI (Figs. 1 and 2). RPE65 levels following IDLV3-RPE65 transduction are approximately 8- to 15-fold higher than control in IDLV3-RPE65transduced cells, whereas in ILV3-RPE65transduced cells, RPE65 levels are 27- to 30-fold higher than control. This is consistent with previously reported findings that IDLVs do not transduce as efficiently as ILVs. ${ }^{7}$ As maximizing the number of transduced cells is advantageous, methods by which IDLV3-RPE65 transduction of BMDCs could be enhanced were investigated. Here, the focus was primarily on murine BMDCs as

Figure 3. Enhancing the infection of BMDCs with IDLV3-RPE65. Murine (Lin-/Sca1+) or human (CD34+) BMDCs were infected ILV3-RPE65 or IDLV-RPE65 (at a $\mathrm{MOI}$ of 50 (A, C-G), 100 (A), or 500 (A), or with $20 \mu \mathrm{L}$ concentrated viral supernatant (B). RetroNectin at $2 \mu \mathrm{g} / \mathrm{cm}^{2}$ was used for all transductions, and was either used by itself $(\mathbf{A}-\mathbf{C})$ or with preloading of vectors $(\mathbf{D}-\mathbf{G})$ or cells $(\mathbf{F})$. Cells were infected for $12 \mathrm{~h}$ and harvested for analysis at $16 \mathrm{~h}$ unless spinoculated (G), where cells were infected for $2 \mathrm{~h}$ at $150 \mathrm{~g}$ and harvested for analysis at $4 \mathrm{~h}$. (A) Increasing the MOI of ILV3-RPE65 increased RPE65 mRNA levels from $\sim 27$-fold over control $(p<0.05)$ at $\mathrm{MOI} 50$ to $\sim 68$-fold over control $(p<0.05)$ at $\mathrm{MOI} 100$ and $\sim 150$-fold over control $(p<0.05)$ with $\mathrm{MOI} 500$. Increasing the MOI of IDLV3-RPE65 did had no significant effect on expression, with RPE65 levels remaining at around sevenfold over control ( $p<0.05)$. (B) Centrifugation of IDLV3-RPE65 supernatant for $2 \mathrm{~h}$ increased recovery of virions, with a $\sim 10$-fold increase in infection of murine BMDCs with concentrated supernatant at the same volume in comparison to eightfold observed at $1 \mathrm{~h}$. Centrifugation for $3 \mathrm{~h}$ did not enhance infection efficiency, and centrifuging for $4 \mathrm{~h}$ reduced the viability of virions, with RPE65 expression only threefold over control $(p<0.05)$. (C) Concentrating viral supernatant $20 \times$ enhanced the infection of IDLV3-RPE65 in comparison with concentrating $10 \times$ and infecting at the same MOI, with a 19-fold $(p<0.05)$ increase in RPE65 mRNA observed at a MOI of 50 with the $10 \times$ concentrated vector and 10 -fold $(p<0.05)$ with the $20 \times$ concentrated vector at the same MOI. (D) Preloading ILV3-RPE65 on RetroNectin for 30 min prior to adding murine BMDCs did not enhance infection, with a 28 -fold increase $(p<0.05)$ observed with or without preloading. In contrast, with IDLV3-RPE65, preloading increased RPE65 expression from 15 -fold over control $(p<0.05)$ to 23 -fold over control $(p<0.05)$. (E) Preloading ILV3-RPE65 on RetroNectin up to three times did not result in an increase in human RPE65 expression. Preloading IDLV3-RPE65 twice increased infection, with RPE65 mRNA levels increasing from 21- to 24-fold over control $(p<0.05)$. No increase was observed with three preloads. (F) Murine BMDCs transduced with IDLV-RPE65 expressed RPE65 at the highest level when viral particles were preloaded onto RetroNectin, with a 24 -fold increase $(p<0.05)$ in expression versus 10 -fold with no preload $(p<0.05)$. Preloading the cells instead of the vector yielded an increase in RPE65 expression, with a 17-fold increase versus control $(p<0.05)$. However, this was lower than observed when viral particles were preloaded, indicating no advantage in preloading cells (G) IDLV-RPE65 infects murine BMDCs at the highest efficiency when the vector is preloaded onto RetroNectin followed by infection by spinoculation, with an 26-fold increase $(p<0.05)$ in expression over control in comparison to a 20 -fold increase with preload alone $(p<0.05)$, and a 22-fold increase with spinoculation alone $(p<0.05)$. $\left({ }^{*} p<0.05\right.$ [SEM]; $\left.n=3\right)$. All statistical significance was calculated using one-way ANOVA followed by Tukey's multiple comparisons test. 

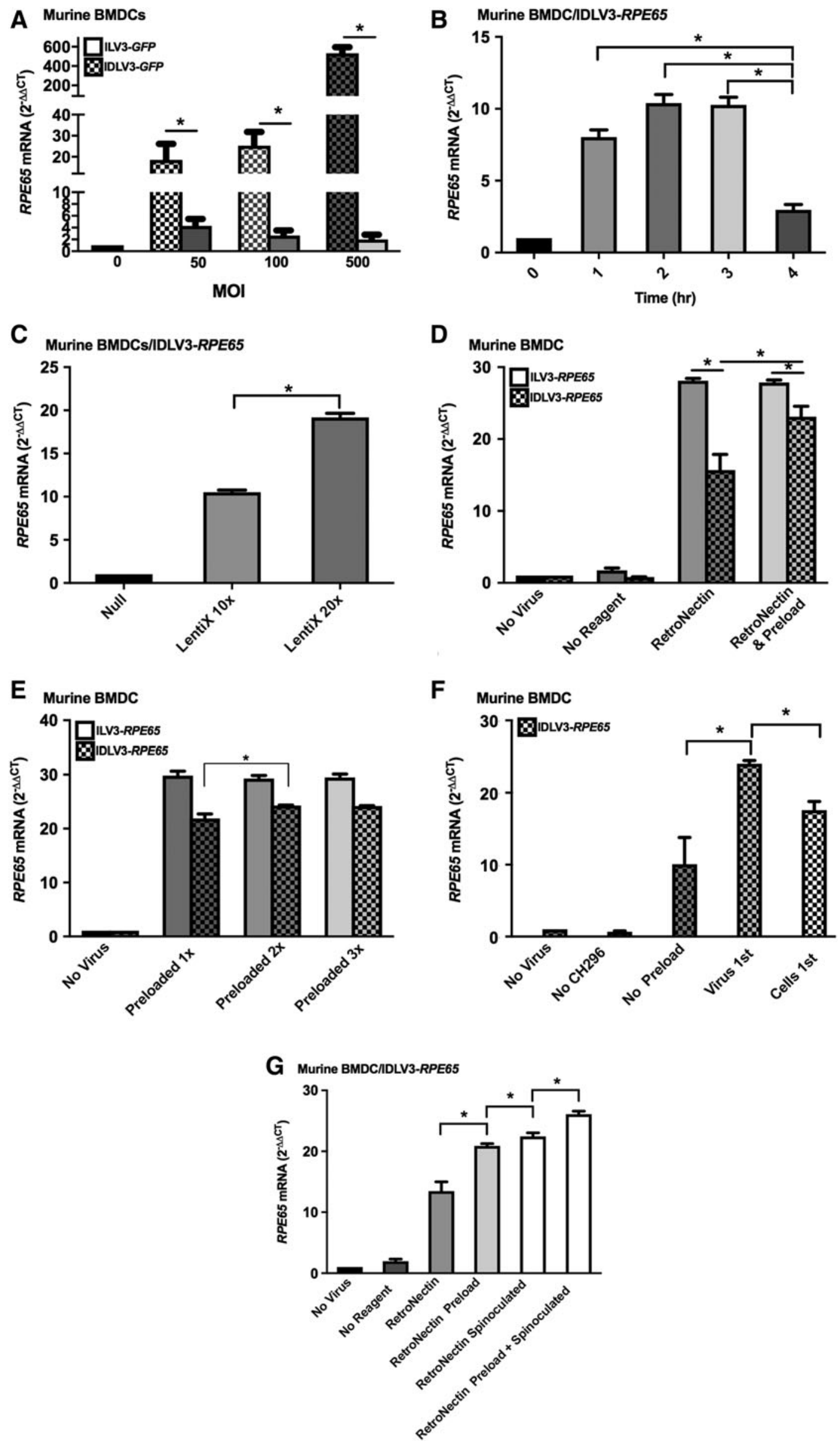
opposed to human BMDCs due to the necessity of establishing the use of IDLV3-RPE65 therapeutically in murine BMDCs prior to testing in our mouse model of retinal degeneration.

Increasing the MOI does not significantly enhance expression in murine BMDCs cells transduced with IDLV3-RPE65, with RPE65 expression remaining at around two- to threefold over control in IDLV3-RPE65-transduced cells, despite an increase of the MOI from 50 to 500 (Fig. 3A). In contrast, increasing the MOI of ILV3-RPE65 from 50 to 500 significantly enhances RPE65 expression, with a $\sim 18$-fold $(p<0.05)$ increase over the control with MOI 50, and a 500-fold increase at MOI 500 $(p<0.05)$. As increasing the MOI did not enhance IDLV transduction, next the procedure by which the viral particles were concentrated was modified. Increasing the centrifugation time from 1 to $2 \mathrm{~h}$ increased the virion recovery of IDLV3-RPE65, with an increase in human RPE65 in cells transduced with $20 \mu \mathrm{L}$ of supernatant from around eightfold over control at $1 \mathrm{~h}$ to $\sim 12$-fold over control at $2 \mathrm{~h}$ (Fig. 3B). Further increasing the concentration time to $3 \mathrm{~h}$ did not enhance virion recovery. Additionally, centrifuging the viral particles for $4 \mathrm{~h}$ significantly reduced recovery, with RPE65 expression observed at less than fivefold over the control in BMDCs transduced with $20 \mu \mathrm{L}$ of IDLV3RPE65 supernatant.

To investigate further whether the concentration protocol could be modified to enhance IDLV3RPE65 transduction, the viral supernatant was concentrated with LentiX Lentiviral Vector Concentrating Reagent (catalog \#PT4421-2; ClonTech). Concentrating the viral supernatant $10 \times$ with LentiX resulted in a $\sim 12$-fold $(p<0.05)$ increase in expression over the control when transduced at an MOI of 50. IDLV3-RPE65 concentrated at $20 \times$ resulted in a significant increase in RPE65, with a $\sim 20$-fold $(p<0.05)$ increase at the same MOI (Fig. 3C).

While Lenti-X concentration of IDLV3-RPE65 enhanced transduction efficiency in comparison with the centrifugation-concentrated vector, the efficiency was still lower than that of ILV3-RPE65, with a $\sim 20$-fold increase with IDLV3-RPE65 compared to an average 27-fold increase with ILV3$R P E 65$. It has been reported that preloading retroviral vectors onto RetroNectin, either once or up to five times, significantly enhances gene transfer. ${ }^{12}$ Therefore, the use of preloading in ILV3-RPE65 and IDLV3-RPE65 transductions was evaluated. RPE65 levels after ILV3-RPE65 transduction remained at $\sim 27$-fold $(p<0.05)$ over control when murine BMDCs were transduced with or without preloading (Fig. 3D). In contrast, RPE65 expression increased from $\sim 13$-fold $(p<0.05)$ over control on RetroNectin alone to $\sim 23$-fold $(p<0.05)$ in murine BMDCs transduced with IDLV3-RPE65 preloaded on the RetroNectin (Fig. 3D). Preloading the plate twice enhances IDLV3-RPE65 transduction ( $p<0.05$; Fig. 3E). No significant improvement was observed between two and three preloads (Fig. 3E).

As RetroNectin binds to both the vector and the cells, transduction on plates that had been preloaded with cells prior to the addition of the vector was also considered. No advantage in preloading cells in comparison with preloading viral supernatant was observed (Fig. 3F).

Despite the increase in IDLV3-RPE65 transduction with preloading, RPE65 levels were still lower ( $\sim 23$-fold over control) than those obtained with ILV3-RPE65 ( 27-fold over control). As a final modification to the protocol, RetroNectin preloading was combined with transduction by centrifugation, referred to as spinoculation. Spinoculating murine BMDCs with preloaded IDLV3-RPE65 results in $\mathrm{a} \sim 27$-fold increase in expression of the vector when combined with preloading on RetroNectin without spinoculation $(p<0.05$; Fig. $3 \mathrm{G})$.

The study therefore demonstrated that concentrating IDLV3-RPE65 $20 \times$ with LentiX followed by transduction of cells on $2 \mu \mathrm{g} / \mathrm{cm}^{2}$ RetroNectin, preloaded with the vector at a MOI of 50, with spinoculation, increases RPE65 expression at a similar level to ILV3-RPE65.

\section{In vitro differentiation of human and murine BMDCs with IDLV3-RPE65}

It was previously reported that transduction of murine BMDCs with an integrating lentiviral vector expressing $R P E 65$ initiates expression of the endogenous murine Rpe65 and Cralbp mRNAs (Fig. 1A). ${ }^{1,2}$ Murine BMDCs transduced with either ILV3-RPE65 (Fig. 1A) or IDLV3-RPE65 vectors (Fig. 4A) express endogenous Rpe65, and Cralbp mRNAs 5- to 10 -fold $(p<0.05)$ over control (Fig. 4A). This indicates that IDLV3-RPE65 is likely to be sufficient for promoting the expression of the RPE-associated genes that contribute to the programming of the BMDCs to RPE-like cells. In human BMDCs, an approximately fivefold increase in expression of CRALBP mRNA is observed in cells transduced with either ILV3-RPE65 or IDLV3-RPE65 (Fig. 4B). This indicates that IDLV3-RPE65 may also be capable of promoting differentiation of human BMDCs to RPE-like cells. While the relative contribution of the exogenous (i.e., vector-derived) RPE65 in comparison to the 

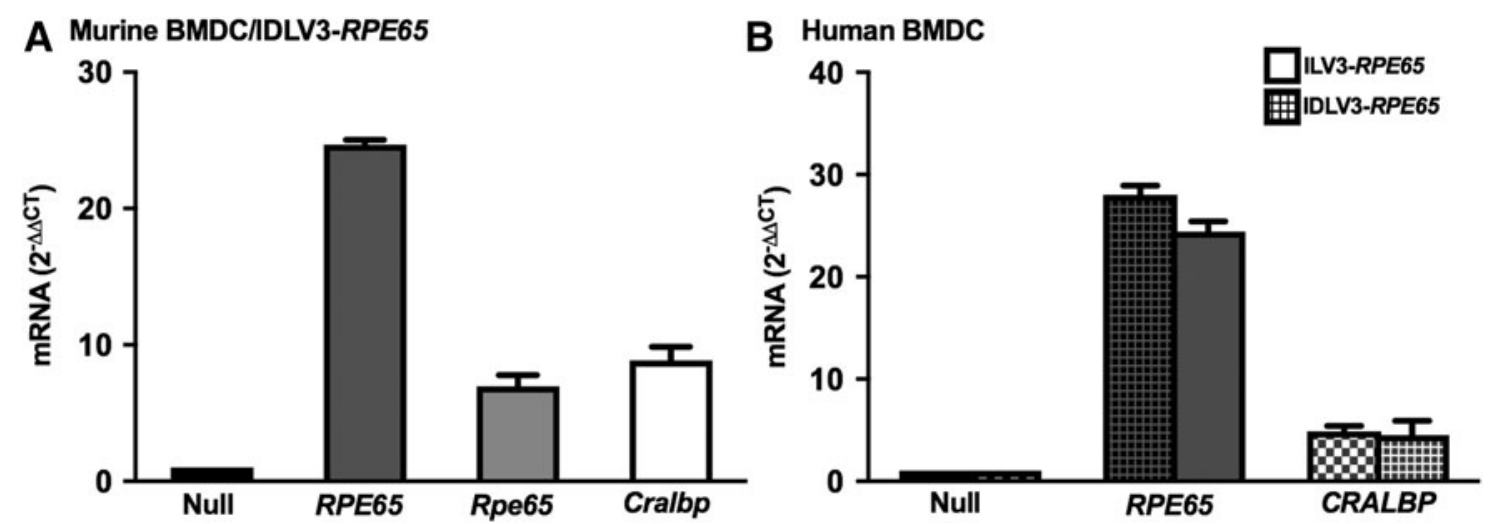

Figure 4. In vitro differentiation of human and murine BMDCs with IDLV3-RPE65. Murine Lin-/Sca1+ BMDCs (A) or human CD34+ cells (B) were transduced with ILV3-RPE65 on RetroNectin by spinoculation for $2 \mathrm{~h}$ at $150 \mathrm{~g}$, or with IDLV3-RPE65 preloaded on RetroNectin prior to spinoculation. Cells were then harvested and lysed for qRT-PCR analysis. RPE65 expression was 32-fold over control and $\sim 28$-fold over control in murine (A) and human (B) BMDCs infected with ILV3-RPE65, respectively $(p<0.05)$. RPE65 mRNA levels were $\sim 24$-fold over control in both murine (A) and human (B) BMDCs, respectively $(p<0.05)$. In murine BMDCs, endogenous Rpe65 mRNA levels were increased around six- and sevenfold versus control in cells transduced with ILV3-RPE65 and IDLV3-RPE65, respectively, and Cralbp mRNA levels were increased seven- and eightfold versus control in cells transduced with ILV3-RPE65 and IDLV3$R P E 65$, respectively (A). In human BMDCs, CRALBP mRNA levels were fivefold over control in cells transduced with either vector. $\left(^{*} p<0.05\right.$ [SEM]; $n=12$ [murine BMDC, ILV3-RPE65]; $n=3$ [human BMDC, ILV3-RPE65; murine and human BMDC, IDLV3-RPE65]). All statistical significance was calculated using oneway ANOVA followed by Tukey's multiple comparisons test.

endogenous Rpe65 gene is not currently known, it is proposed that activation of the endogenous Rpe65 is likely to be critical for adequate cellular reprogramming. It was observed that $R p e 65$ activation also results in a lack of Cralbp activation in murine BMDCs, suggesting a role for Rpe 65 versus $R P E 65$ specifically in programming the BMDCs.

\section{Efficacy of ILV3 and IDLV3 in a murine model of retinal degeneration}

Rescue of visual function can be assessed using the optokinetic response, which is determined by measuring the spatial frequency of head turning in response to an image. The optokinetic response was greatly reduced following SOD2 knockdown compared to control mice (Fig. 5A), and this was not improved by systemic administration of naïve BMDC. However, a significant improvement $(>60 \% ; p<0.05)$ in optokinetic response was observed in animals 3 months after receiving systemic BMDC-ILV3-RPE65 or BMDC-IDLV3RPE65 (Fig. 5A). OCT assessment demonstrated normal retinal architecture in untreated eyes. However, SOD2 knockdown resulted in significant abnormalities in SOD2 eyes at 4 months following knockdown, as reported previously, ${ }^{1}$ and this was not reversed by the systemic administration of naïve BMDCs (Fig. 5B). By contrast, animals 3 months after receiving systemic BMDC-ILV3RPE65 or BMDC-IDLV3-RPE65 demonstrated near normal retinal architecture (Fig. 5B). In agreement with previous observations, ${ }^{1} \mathrm{RPE}$ flat mounts demonstrated the typical appearance of $\mathrm{GFP}^{+} \mathrm{BMDCs}$ in mice receiving systemic BMDCILV3-RPE65 or BMDC-IDLV3-RPE65 (Fig. 5C). Only minimal numbers of $\mathrm{GFP}^{+} \mathrm{BMDCs}$ were observed in animals receiving naïve BMDCs. Pathology in the SOD2 knockdown retinas demonstrated vacuolation and atrophy of the RPE together with degeneration of the photoreceptors (Fig. 5D). Mice treated with control naïve BMDCs demonstrated similar progressive pathology to that of untreated SOD2 knockdown mice. By contrast, mice receiving systemic BMDC-ILV3-RPE65 or BMDC-IDLV3-RPE65 demonstrated a mature, highly pigmented epithelial layer, with RPE morphology consistent with the normal controls, and the morphology of the neural retina was greatly improved (Fig. 5D).

\section{DISCUSSION}

It was previously shown in two murine models of RPE damage that adult BMDCs modified ex vivo with an ILV expressing RPE65 migrate to and preserve the RPE and retina following systemic delivery. ${ }^{1,2}$ Compounding factors facilitating the lack of visual recovery in humans after cell-based therapy for dry AMD to date ${ }^{15-17}$ include treatment in the late stages of disease, limited motility of cells across the RPE layer post-injection, and side effects of invasive cell delivery. ${ }^{1,2,18-20}$ The systemicdelivery approach eliminates the need for invasive sub-retinal surgery, allowing for early intervention. The cells are more likely to integrate across 

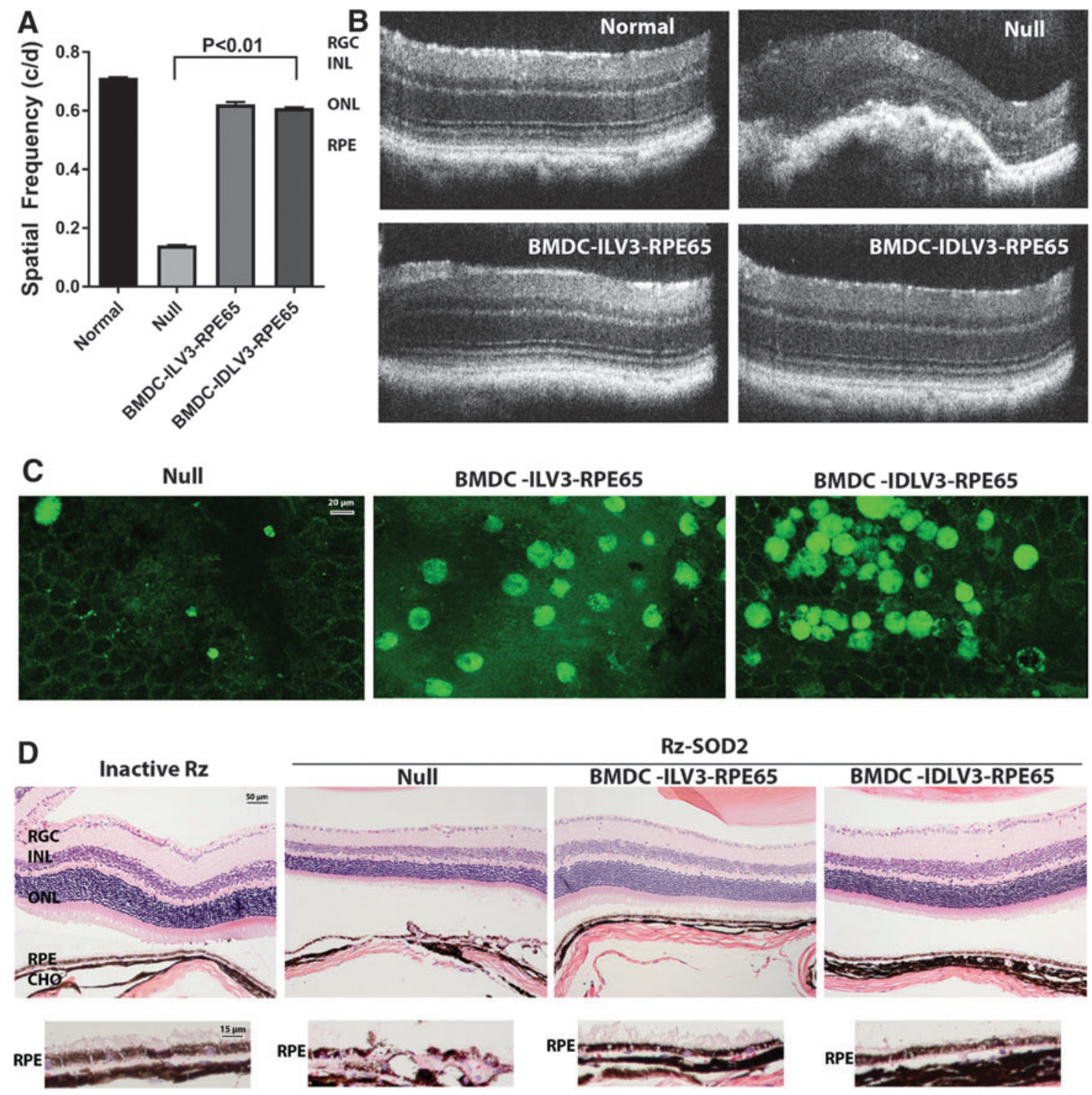

Figure 5. Efficacy of ILV3 and IDLV3 programmed BMDCs in a murine model of retinal degeneration. C57BL6/J mice received sub-retinal injection of AAV1-RzSOD2 or AAV1-Rz-inactive 1 month prior to tail-vein injections of naïve GFP ${ }^{+}$murine BMDCs (null) or GFP ${ }^{+}$BMDCs programmed with ILV3-RPE65 or IDLV3-RPE65. Animals were assessed 3 months following administration of BDMCs. (A) Visual acuity testing using an Optomotor behavioral test demonstrated recovery of optokinetic response in SOD2 knockdown mice receiving ILV3-RPE65-programmed BMDCs or IDLV3-RPE65-programmed BMDCs, which was not observed in animal receiving naïve BMDCs (null). A comparison of average values for photopic activities in response to rotating sinusoidal gratings is shown for each group of mice ( $n=5$ per group) as mean \pm SEM. (B) Spectral domain optical coherence tomography (SD-OCT) was used to examine retinal morphology of the mouse retina in vivo. Major abnormalities were observed in the RPE/photoreceptor layer in RzSOD2 knockdown eyes with or without null BMDC treatment. However, the RzSOD2 knockdown eyes that received the ILV3-RPE65-programmed BMDC or IDLV3-RPE65-programmed BMDC treatments show near-normal retina architectures. (C) Representative fluorescent micrographs of flat-mounted RPE showing the presence of significant numbers of GFP ${ }^{+}$(green) IDLV3-RPE65-BMDC and ILV3-RPE65BMDCs. Only very few $\mathrm{GFP}^{+}$cells were observed in mice treated systemically with null BMDCs. (D) Representative photomicrographs of $6 \mu \mathrm{M}$ hematoxylin and eosin-stained cross-sections of the retina/choroid/sclera showed significant disruption of the RPE layer in the SOD2-KD mice, which was largely absent in mice receiving either ILV3-RPE65-programmed BMDC or IDLV3-RPE65-programmed BMDC, which both showed much improved retinal morphology. Higher magnification images of the RPE layer demonstrated that systemic administration of RPE65-programmed BMDCs with either ILV3 or IDLV3 have a near normal RPE morphology, similar to inactive Rz injected eyes. This was not apparent in animals receiving null BMDCs, in which there is thinning of the retina and severe loss of the RPE cells. RGC, retinal ganglion cell layer; INL, inner nuclear layer; ONL, outer nuclear layer; RPE, retinal pigment epithelium; CHO, choroid.

the RPE layer and at focal points of injury due to their recruitment from the bloodstream. ${ }^{21,22}$

Transient expression of RPE65 is likely to be sufficient, as it was previously reported that the endogenous Rpe65 and Cralbp genes are activated within hours of RPE65 vector transduction. ${ }^{1,2}$ IDLVs lack the integrase gene, and integrate at a 500- to 1,250-fold lower rate than integrating vectors, ${ }^{13}$ with residual integration likely occurring through integrase-independent mechanisms, ${ }^{23}$ 
such as DNA break-induced non-LTR-mediated integration. ${ }^{24}$ In a clinical setting, inclusion of a suicide gene in the transducing vector would allow for rapid elimination of cells containing integrated vector if off-target effects resulting from insertional mutagenesis were to occur. Suicide gene-mediated inducible apoptosis has been demonstrated to be safe and effective in human clinical trials. ${ }^{25,26}$ The findings are consistent with observations that low-level transgene expression is maintained 10-14 days post transduction. ${ }^{27-29}$ Residual integration is, however, not considered to be a significant concern, considering that even integrating vectors that have been modified to self-inactivate, as is now standard practice for the use of LV vectors, do not promote oncogenesis, even in murine models that are tumor prone. ${ }^{30}$

It has been well established in the literature that IDLVs are less efficient than ILVs. ${ }^{6,7}$ Critical difficulties in the application of IDLVs have arisen for a number of reasons, primarily a low number of virions entering cells, ${ }^{6}$ and downregulation of transgene expression due to episome inhibition, ${ }^{11}$ which has been shown to be counteracted by a deletion in the U3 region of the vector. ${ }^{11}$ Several groups have published modifications to the transducing vector which improve the stability of expression from episomal IDLV particles. These modifications include codon-optimization of the transgene, ${ }^{31,32}$ the use of a strong promoter such as $\mathrm{SV} 40^{33}$ instead of the CMV promoter, which is susceptible to silencing, ${ }^{34}$ and inhibiting viral lifecycle proteins. ${ }^{35,36}$ Some of these modifications have been utilized in this study through the use of the EF-1 promoter, which is constitutively expressed at a similar level in all cell types, and codon-optimization of the RPE65 cDNA for use in human cells.

Overall, the focus was on increasing the number of virions entering cells as opposed to modifying the transducing vector further, as retention of transgene expression was not thought to be required for the application. The critical difference between the use of IDLV3-RPE65 in this study and the majority of IDLV3-based studies published to date is that expression of RPE65 in this study was only required at a high level for a few hours post transduction for the vector to activate the endogenous genes, and therefore it was being used as a molecular "switch" as opposed to a long-term modification. Consequently, expression was measured at $4 \mathrm{~h}$ post transduction, which is in contrast with typical LV expression studies that measure transgene expression after several days in culture..$^{, 7,11,13,29,32,33,36-45}$ This time point was chosen, as it has previously been demonstrated that $R P E 65$ induces Rpe65 and Cralbp expression within the first few hours of transduction. ${ }^{2}$

It is proposed that the method outlined here is optimal for the application of IDLVs in studies in which short-term (1-12 h) expression is sufficient, as the increase in expression in the early stages post transduction may not be sustained. The success in improving expression through increasing viral number per cell is likely to precede silencing of the episome. ${ }^{11}$ For studies in which longer-term expression of the transgene is required, vector modifications such as U3 deletions may be necessary to maintain transgene expression after increasing the transduction efficiency. ${ }^{11}$ Long-term expression from IDLVs has, however, been demonstrated in several studies, primarily in the liver, in which coagulation Factor IX has been shown to be stably expressed from IDLVs in levels comparable to those observed with ILVs. ${ }^{46,47}$ YanezMunoz et al. have also shown robust long-term expression of an IDLV-derived transgene following in vivo application of a GFP-expressing IDLV in the brain and retinae of mice. ${ }^{48}$ In vivo transduction of RPE cells with the RPE65 vector is not a feasible treatment for dry AMD, as cell replacement, rather than gene replacement, is required.

To improve IDLV3-RPE65 transduction efficiency, the transduction protocol was systematically modified. Transduction is dependent on direct virion-cell interaction. Viral particles have a halflife of around $4-8 \mathrm{~h},{ }^{49,50}$ during which they are capable traveling 580-610 microns. ${ }^{51}$ Since both the cells and the vectors are negatively charged, it is necessary to enhance the localization of cells and virions using reagents that either bind both, or eliminate the negative charge of both the cells and the virions. RetroNectin enhances transduction efficiency via co-localization of virions and cells ${ }^{52,53}$ through binding by a heparin-binding domain and VLA5/VLA4 binding domains respectively. ${ }^{12,14}$ RetroNectin is frequently used to maintain retroviral gene transfer in hematopoietic stem cells derived from primates, canines, and humans. ${ }^{23,54,55}$ It has advantages over the commonly used polycation polybrene, which facilitates gene transfer by counteracting the negative charge of the cells and virions, in that it is less toxic to cells yet produces similarly efficient transductions. Protamine sulfate has been shown to be as effective as polybrene with the mouse SAX retroviral vector. ${ }^{56}$ However, it was found to be significantly less effective than polybrene or RetroNectin in BMDCs. Therefore, the focus was on using RetroNectin to enhance transduction with IDLV3-RPE65. 
Efficient transduction of the HEL cell line and human CD34+ cells with retroviral vectors has been reported on plates coated with $2 \mu \mathrm{g} / \mathrm{cm}^{2}$ RetroNectin, ${ }^{12}$ despite the instruction manual indicating a requirement for $4-20 \mu \mathrm{g} / \mathrm{cm}^{2}$. The findings support these data, indicating that $\mathrm{LVs}$ also transduce both human and mouse BMDCs on $2 \mu \mathrm{g} /$ $\mathrm{cm}^{2}$ RetroNectin. Additionally, the study shows that a single preparation of $2 \mu \mathrm{g} / \mathrm{cm}^{2}$ RetroNectin can be removed and used to coat up to three wells before any reduction in efficiency. Both of these findings minimize the cost of LV approaches using RetroNectin. Also consistent with the literature, in which it has been reported that preloading of retroviral vectors on RetroNectin enhances gene transfer, ${ }^{12}$ preloading of IDLV on RetroNectin prior to transduction of cells resulted in a higher transduction efficiency, ${ }^{12}$ indicating that preloading is effective for transduction with lentiviral as well as retroviral vectors.

Expression of the endogenous Rpe65 and Cralbp genes in murine BMDCs transduced with IDLV3-RPE65 confirm that the IDLV is functioning in a similar way to the ILV. To confirm this, it was demonstrated that systemic administration of BMDCs transduced with IDLV3-RPE65 or ILV3-RPE65 both resulted in significantly improved visual function in a mouse model of retinal degeneration that mimics many of the features of early AMD. ${ }^{57}$ Furthermore, in agreement with previous studies, ${ }^{1,2}$ the BMDCs were recruited to the RPE layer and reduced retinal degeneration in comparison to mice receiving treatment with naïve BMDC. An in-depth phenotypic analysis of the donor cells was not undertaken in this study, as the primary endpoint was retention of visual function. However, it has been previously shown that these RPE65-programmed BMDCs are recruited to the host RPE monolayer and take on an RPE-like phenotype, including phagocytosis of photoreceptor outer segments., ${ }^{1,2}$ In addition, these cells may play a neuroprotective role in the degenerating retina.

Expression of CRALBP in human BMDCs transduced with ILV3-RPE65 or IDLV3-RPE65 indicates that expression of RPE65 in these cells is functioning in a similar way as expression in murine BMDCs, and suggests that expression of this is likely to result in human BMDC differentiation into RPE-like cells. This is of significant importance for the therapeutic potential of this technique in humans, which will be the focus of future studies.

To summarize, use of IDLV3-RPE65 significantly reduces the risk of the LV-based approach, as the number of viral integrations per cell and the subsequent risk of insertional mutagenesis is markedly reduced. IDLV3-RPE65 successfully initiates the expression of endogenous genes, which is believed to be responsible for the ability of RPE65-transduced BMDCs to integrate into and regenerate damaged RPE in mouse models of RPE degeneration in both murine and human BMDCs. Efficacy is significantly enhanced by modifying the transduction protocol with RetroNectin. This approach is quick and ideal for applications in which short-term transient expression may be sufficient, avoiding time-consuming and costly modifications of the transducing vector.

\section{ACKNOWLEDGMENTS}

We thank Drs. Kenneth Cornetta and Aaron Shaw (IU School of Medicine) and Dirk Lindemann (Technische Universitat Dresden) for providing plasmids for lentiviral vector production, Drs. David Gilley, Scott Witting, and Kenneth Cornetta (IU School of Medicine) for providing cell lines, Dr. Helmut Hanenberg (Heinrich Heine Universitat Dusseldorf) for plasmids and advice on the use of RetroNectin for lentiviral vector transduction and Dr. Alfred Lewin (University of Florida) for help with the SOD2 knockdown mouse model. This paper was previously submitted as part of the graduation requirements for a $\mathrm{PhD}$ at Indiana University undertaken by S.L.P. This research was supported by the NIH (EY023629), NEI Core grant P30 EY03039, BrightFocus (M2009024), and an unrestricted grant from Research to Prevent Blindness.

\section{AUTHOR DISCLOSURE}

No competing financial interests exist.

\section{REFERENCES}

1. Qi $X$, Pay SL, Yan $Y$, et al. Systemic injection of RPE65-programmed bone marrow-derived cells prevents progression of chronic retinal degeneration. Mol Ther 2017;25:917-927.
2. Sengupta N, Caballero S, Sullivan SM, et al. Regulation of adult hematopoietic stem cells fate for enhanced tissue-specific repair. Mol Ther 2009; 17:1594-1604.
3. Wong WL, Su X, Li X, et al. Global prevalence of agerelated macular degeneration and disease burden projection for 2020 and 2040: a systematic review and meta-analysis. Lancet Glob Health 2014;2:e106-116. 
4. Bird AC. Pathogenic mechanisms in age-related macular degeneration. In: Ryan SJ, ed. Ryan's Retina. 5th ed. Amsterdam: Elsevier, 2013:11451149

5. Maier $P$, von Kalle $C$, Laufs $S$. Retroviral vectors for gene therapy. Future Microbiol 2010;5:15071523

6. Joglekar AV, Hollis RP, Kuftinec G, et al Integrase-defective lentiviral vectors as a delivery platform for targeted modification of adenosine deaminase locus. Mol Ther 2013;21:1705-1717.

7. Shaw AM, Joseph GL, Jasti AC, et al. Differences in vector-genome processing and illegitimate integration of non-integrating lentiviral vectors. Gene Ther 2017;24:12-20.

8. Jin C, Fotaki G, Ramachandran M, et al. Safe engineering of CAR T cells for adoptive cell therapy of cancer using long-term episomal gene transfer. EMBO Mol Med 2016;8:702711.

9. Ranzani M, Annunziato S, Adams DJ, et al. Cancer gene discovery: exploiting insertional mutagenesis. Mol Cancer Res 2013;11:1141-1158.

10. Schambach A, Zychlinski D, Ehrnstroem B, et al. Biosafety features of lentiviral vectors. Hum Gene Ther 2013;24:132-142.

11. Bayer M, Kantor B, Cockrell A, et al. A large U3 deletion causes increased in vivo expression from a nonintegrating lentiviral vector. Mol Ther 2008; 16:1968-1976.

12. Hanenberg $\mathrm{H}$, Hashino $\mathrm{K}$, Konishi $\mathrm{H}$, et al. Optimization of fibronectin-assisted retroviral gene transfer into human CD34+ hematopoietic cells. Hum Gene Ther 1997;8:2193-2206.

13. Philippe S, Sarkis C, Barkats M, et al. Lentiviral vectors with a defective integrase allow efficient and sustained transgene expression in vitro and in vivo. Proc Natl Acad Sci U S A 2006;103: 17684-17689

14. Dutt $P$, Hanenberg $H$, Vik T, et al. A recombinant human fibronectin fragment facilitates retroviral mediated gene transfer into human hematopoietic progenitor cells. Biochem Mol Biol Int 1997;42 909-917.

15. Schwartz SD, Hubschman JP, Heilwell G, et al Embryonic stem cell trials for macular degeneration: a preliminary report. Lancet 2012;379:713720 .

16. Schwartz SD, Regillo CD, Lam BL, et al. Human embryonic stem cell-derived retinal pigment epithelium in patients with age-related macular degeneration and Stargardt's macular dystrophy: follow-up of two open-label Phase 1/2 studies. Lancet 2015;385:509-516

17. Mandai $M$, Watanabe $A$, Kurimoto $Y$, et al. Autologous induced stem-cell-derived retinal cells for macular degeneration. N Engl J Med 2017; 376:1038-1046.

18. Kamao H, Mandai M, Okamoto $S$, et al. Characterization of human induced pluripotent stem cell-derived retinal pigment epithelium cell sheets aiming for clinical application. Stem Cell Reports 2014;2:205-218.

19. Qi X, Pay SL, Yan Y, et al. Systemic injection of RPE65-programmed bone marrow-derived cells prevents progression of chronic retinal degeneration. Mol Ther 2017:25:917-927.

20. Sengupta N, Caballero S, Mames RN, et al. The role of adult bone marrow-derived stem cells in choroidal neovascularization. Invest Ophthalmol Vis Sci 2003;44:4908-4913.

21. Bhutto IA, McLeod DS, Merges C, et al. Localisation of SDF-1 and its receptor CXCR4 in retina and choroid of aged human eyes and in eyes with age related macular degeneration. $\mathrm{Br} \mathrm{J}$ Ophthalmol 2006;90:906-910.

22. Harris JR, Brown GA, Jorgensen $M$, et al. Bone marrow-derived cells home to and regenerate retinal pigment epithelium after injury. Invest Ophthalmol Vis Sci 2006;47:2108-2113.

23. Kiem HP, Andrews RG, Morris J, et al. Improved gene transfer into baboon marrow repopulating cells using recombinant human fibronectin fragment $\mathrm{CH}-296$ in combination with interleukin-6, stem cell factor, FLT-3 ligand, and megakaryocyte growth and development factor. Blood 1998:92: 1878-1886.

24. Wanisch K, Yanez-Munoz RJ. Integration-deficient lentiviral vectors: a slow coming of age. Mol Ther 2009;17:1316-1332.

25. Di Stasi A, Tey SK, Dotti G, et al. Inducible apoptosis as a safety switch for adoptive cell therapy. N Engl J Med 2011;365:1673-1683

26. Zhou X, Brenner MK. Improving the safety of Tcell therapies using an inducible caspase- 9 gene. Exp Hematol 2016;44:1013-1019.

27. Case SS, Price MA, Jordan CT, et al. Stable transduction of quiescent CD34(+)CD38(-) human hematopoietic cells by HIV-1-based lentiviral vectors. Proc Natl Acad Sci U S A 1999;96:29882993.

28. Haas DL, Case SS, Crooks GM, et al. Critical factors influencing stable transduction of human CD34(+) cells with HIV-1-derived lentiviral vectors. Mol Ther 2000;2:71-80.

29. Vargas J Jr, Gusella GL, Najfeld V, et al. Nove integrase-defective lentiviral episomal vectors for gene transfer. Hum Gene Ther 2004;15:361-372.

30. Montini E, Cesana D, Schmidt M, et al. Hematopoietic stem cell gene transfer in a tumor-prone model uncovers low genotoxicity of lentiviral vector integration. Nat Biotechnol 2006;24:687696.

31. Suwanmanee T, Hu G, Gui T, et al. Integrationdeficient lentiviral vectors expressing codonoptimized R338L human FIX restore normal hemostasis in Hemophilia B mice. Mol Ther 2014;22: 567-574.

32. Negri DR, Michelini Z, Baroncelli S, et al. Successful immunization with a single injection of non-integrating lentiviral vector. Mol Ther 2007; 15:1716-1723.
33. Vargas J Jr, Klotman ME, Cara A. Conditionally replicating lentiviral-hybrid episomal vectors for suicide gene therapy. Antiviral Res 2008;80: 288-294.

34. Teschendorf C, Warrington KH Jr, Siemann DW et al. Comparison of the EF-1 alpha and the CMV promoter for engineering stable tumor cell lines using recombinant adeno-associated virus. Anticancer Res 2002;22:3325-3330.

35. Berger G, Goujon C, Darlix JL, et al. SIVMAC Vpx improves the transduction of dendritic cells with nonintegrative HIV-1-derived vectors. Gene Ther 2009:16:159-163.

36. Negri DR, Rossi A, Blasi M, et al. Simian immunodeficiency virus-Vpx for improving integrase defective lentiviral vector-based vaccines. Retrovirology 2012;9:69.

37. Lombardo A, Genovese P, Beausejour CM, et al. Gene editing in human stem cells using zinc finger nucleases and integrase-defective lentiviral vector delivery. Nat Biotechnol 2007;25 1298-1306.

38. Blomer U, Naldini L, Kafri T, et al. Highly efficient and sustained gene transfer in adult neurons with a lentivirus vector. J Virol 1997;71:6641-6649.

39. Mochizuki H, Schwartz JP, Tanaka K, et al. Hightiter human immunodeficiency virus type 1-based vector systems for gene delivery into nondividing cells. J Virol 1998;72:8873-8883.

40. Yung E, Sorin M, Pal A, et al. Inhibition of HIV-1 virion production by a transdominant mutant of integrase interactor 1. Nat Med 2001;7:920-926

41. Englund G, Theodore TS, Freed EO, et al. Integration is required for productive transduction of monocyte-derived macrophages by human immunodeficiency virus type 1. J Virol 1995;69: 3216-3219

42. Wang $Y$, Wang $Y$, Chang $T$, et al. Integrationdefective lentiviral vector mediates efficient gene editing through homology-directed repair in human embryonic stem cells. Nucleic Acids Res 2017:45:e29.

43. Zhu K, Dobard C, Chow SA. Requirement for integrase during reverse transcription of human immunodeficiency virus type 1 and the effect of cysteine mutations of integrase on its interactions with reverse transcriptase. J Virol 2004;78:50455055

44. Rio P, Banos R, Lombardo A, et al. Targeted gene therapy and cell reprogramming in Fanconi anemia. EMBO Mol Med 2014:6:835-848.

45. Cornu TI, Cathomen T. Targeted genome modifications using integrase-deficient lentiviral vectors. Mol Ther 2007;15:2107-2113

46. Matrai J, Cantore A, Bartholomae CC, et al Hepatocyte-targeted expression by integrasedefective lentiviral vectors induces antigenspecific tolerance in mice with low genotoxic risk. Hepatology 2011;53:1696-1707.

47. Cantore A, Nair N, Valle PD, et al. Hyperfunctional coagulation Factor IX improves the efficacy of 
gene therapy in hemophilic mice. Blood 2012;120: 4517-4520.

48. Yanez-Munoz RJ, Balaggan KS, MacNeil A, et al. Effective gene therapy with nonintegrating lentiviral vectors. 2006;12:348-353.

49. Paul RW, Morris D, Hess BW, et al. Increased viral titer through concentration of viral harvests from retroviral packaging lines. Hum Gene Ther 1993:4:609-615

50. Sanes JR, Rubenstein JL, Nicolas JF. Use of a recombinant retrovirus to study post-implantation cell lineage in mouse embryos. EMBO J 1986;5:3133-3142.

51. Chuck AS, Clarke MF, Palsson BO. Retroviral transduction is limited by Brownian motion. Hum Gene Ther 1996;7:1527-1534.
52. Moritz T, Dutt $P$, Xiao $X$, et al. Fibronectin improves transduction of reconstituting hematopoietic stem cells by retroviral vectors: evidence of direct viral binding to chymotryptic carboxyterminal fragments. Blood 1996;88:855-862.

53. Hanenberg $H$, Xiao XL, Dilloo D, et al. Colocalization of retrovirus and target cells on specific fibronectin fragments increases genetic transduction of mammalian cells. Nat Med 1996;2:876-882.

54. Donahue RE, Dunbar CE. Update on the use of nonhuman primate models for preclinical testing of gene therapy approaches targeting hematopoietic cells. Hum Gene Ther 2001;12:607-617.

55. Neff T, Horn PA, Peterson LJ, et al. Methylguanine methyltransferase-mediated in vivo selection and chemoprotection of allogeneic stem cells in a large-animal model. J Clin Invest 2003;112:1581-1588.

56. Cornetta K, Anderson WF. Protamine sulfate as an effective alternative to polybrene in retroviral-mediated gene-transfer: implications for human gene therapy. J Virol Methods 1989; 23:187-194.

57. Justilien V, Pang JJ, Renganathan K, et al. SOD2 knockdown mouse model of early AMD. Invest Ophthalmol Vis Sci 2007:48:4407-4420.

Received for publication May 10, 2016; accepted after revision October 22, 2017.

Published online: November 20, 2017. 